\title{
Single versus split dose polyethylene glycol for bowel preparation in children undergoing colonoscopy: a systematic review and meta-analysis
}

\author{
Jinjin Cao ${ }^{1 \#}$, Weiying Zhang ${ }^{2 \#}$, Jiajie $\mathrm{Hu}^{3 \#}$, Yan Huang ${ }^{4}$, Li Zhao ${ }^{4}$, Rong Cai ${ }^{4}$, Ying $\mathrm{Bao}^{4}, \mathrm{Mei}^{4}{ }^{4}$ \\ ${ }^{1}$ Department of Gastroenterology, Children's Hospital of Nanjing Medical University, Nanjing, China; ${ }^{2}$ Department of Emergency, Children's \\ Hospital of Nanjing Medical University, Nanjing, China; ${ }^{3}$ The Fourth School of Clinical Medicine of Nanjing Medical University, Nanjing, China; \\ ${ }^{4}$ Department of Nursing, Children's Hospital of Nanjing Medical University, Nanjing, China \\ Contributions: (I) Conception and design: J Cao, W Zhang, M Li; (II) Administrative support: J Cao, W Zhang, J Hu, Y Huang; (III) Provision of \\ study materials or patients: J Cao, Y Huang, L Zhao, R Cai; (IV) Collection and assembly of data: J Cao, W Zhang, L Zhao, Y Huang, R Cai; (V) \\ Data analysis and interpretation: J Cao, Y Huang, R Cai, Y Bao, M Li; (VI) Manuscript writing: All authors; (VII) Final approval of manuscript: All \\ authors. \\ "These authors contributed equally to this work. \\ Correspondence to: Mei Li. Department of Nursing, Children's Hospital of Nanjing Medical University, No. 72, Guangzhou Road, Nanjing, China. \\ Email: nrh8809@163.com.
}

Background: Polyethylene glycol (PEG) has been widely used for bowel preparation. However, the efficacy and safety of single and split dose PEG for bowel preparation in children undergoing colonoscopy remain unclear, it is necessary to evaluate the role of single and split dose PEG for bowel preparation in children population.

Methods: PubMed et al. databases up to September 1, 2019 were systematically searched. Randomized controlled trials (RCTs) single and split dose PEG for bowel preparation in children undergoing colonoscopy were included. Based on the heterogeneity, data were synthesized using random-effects or fixed-effects models. Results were expressed as Mantel-Haenszel style odds ratio (OR) or mean difference (MD) with 95\% confidence interval (95\% CI).

Results: Four RCTs with 249 children were included. There was no significantly difference in the efficacy of single and split dose PEG for bowel preparation (OR $=0.36,95 \% \mathrm{CI}-0.12$ to 1.10 ). The acceptability of split dose PEG for bowel preparation was significantly higher than that of single dose (OR $=0.50,95 \% \mathrm{CI}$ : 0.29 to 0.85 ); the incidence of nausea in split dose PEG for bowel preparation was significantly lower than that of single dose ( $\mathrm{OR}=2.1,95 \% \mathrm{CI}$ : 1.29 to 3.42); there was no significant difference on the incidence of abdominal pain between two regimes (OR $=1.39,95 \% \mathrm{CI}: 0.67$ to 2.89 ).

Conclusions: Split dose PEG seems to be more superior to single dose for children undergoing colonoscopy. However, considering that the number of included RCTs are very limited, more related studies on this issue are needed in the future.

Keywords: Polyethylene glycol (PEG); bowel preparation; children; review; meta-analysis

Submitted Mar 08, 2020. Accepted for publication Jul 31, 2020.

doi: $10.21037 / \mathrm{apm}-20-582$

View this article at: http://dx.doi.org/10.21037/apm-20-582

\section{Introduction}

Colonoscopy is one of the most important diagnostic and therapeutic procedures of colon disorders (1-3). Adequate bowel preparation is necessary as bowel imaging is critical in this examination. The success of colonoscopy is determined by high-quality bowel preparation which makes the intestinal mucosa visible, and it helps to diagnose and treat smoothly, also it may minimize the risk of contamination 
during surgery (3-6). The adverse consequences caused by insufficient intestinal cleaning include incomplete visualization, missed lesion detection, reduced safety, prolonged examination time, shortened follow-up interval, and increased economic burden on patients $(4,7)$. In the population of children, the methods of bowel preparation must be selected according to the age, size, and clinical status of patients (8). Adequate bowel preparation is considered to be the most difficult part of the colonoscopy process since children have a much greater problem with agents compared with adults who are more likely to take them. Efficacy, safety and tolerance are all the factors needed to be considered. In clinical practice, the choice of intestinal cleanser depends more on the experience of each medical department. Since the wide differs of bowel preparation, it remains a challenge for pediatric colonoscopy to select an ideal preparation regimen. To date, there are only a few well-controlled studies have been published in the pediatric population.

From the consideration of efficacy, safety profile and ease of usage, polyethylene glycol (PEG) which as an osmotic agent is seemed to be the drug choice. This has been proved in several studies (9-11) and is being proposed as laxative of choice for colon preparation by adult as well as by pediatric guidelines $(3,12)$. The main concern with PEG preparation is that of large volume of fluid that a patient has to drink. In pediatric population, drinking large volume of PEG over a fixed time is further complicated by poor tolerability. Although PEG has been found to be safe in children (13), but it is often associated with nausea, vomiting and abdominal distension (14).

In order to overcome the volume related issues of single dose PEG, the study was commonly conducted in adult population with a divided dose regimen. In split dose regimen, the same dose of PEG is given in half, half the night before and half the morning of the colonoscopy. Multiple randomized controlled trials (RCTs) $(15,16)$ as well as meta-analysis $(17)$, have documented the superiority of split dose over single dose in terms of efficacy and tolerability in adult patients. Split dose PEG is now the choice of preparation for colonoscopy in adults (3). However, the recent meta-analysis in children mainly compare efficacy of various agents but they fail to specifically compare various regimens of the same agent (i.e., PEG) (8), the optimization of using PEG in pediatric population remains unclear.

Therefore, the aim of this systematic review is to evaluate the efficacy and tolerability of split dose as compared to single dose PEG solution in pediatric patients undergoing colonoscopy.

We present the following article in accordance with the PRISMA reporting checklist (available at http://dx.doi. org/10.21037/apm-20-582).

\section{Methods}

\section{Eligibility criteria}

RCTs written in English were included, independent of publication status, publication date, risk of bias, publication results. Trials were included if they enrolled children undergoing colonoscopy while using PEG for bowel preparation. Included studies had to compare single versus split dose regimen of PEG. The primary outcome was the efficacy of bowel preparation. Secondary outcomes were the tolerability and safety of bowel preparation. Adverse effects included nausea, vomiting, abdominal pain, bloating. The common indications for colonoscopy were bloody stool and abdominal pain. Case reports, qualitative studies, and reviews articles were excluded. All studies were screened independently by two authors according to the selection criteria. The studies were selected when the two reviewers consented. Any differences regarding inclusion studies were resolved by a third reviewer. Since our manuscript is a metaanalysis, no ethical permissions were needed. The study conformed to the provisions of the Declaration of Helsinki (as revised in 2013).

\section{Search methods}

MEDLINE, EMBASE, and CENTRAL were searched for keywords describing the condition, intervention, or comparator from inception to September 1, 2019. Searching was conducted through strategies used in PubMed and the instructions and rules of each database. The search terms were as following: "Child" OR "children" AND "Polyethylene Glycols" OR "Macrogol" OR "Polyethylene Oxide" OR "Oxide, Polyethylene" OR "Oxides, Polyethylene" OR "Polyethylene Oxides" OR "Polyethylene oxide" OR "Polyoxymethylene" OR "Polyglycol" OR "Polyethylene Glycol" OR "Glycol, Polyethylene" OR "Glycols, Polyethylene" OR "Carbowax" OR "PEG" AND "Colonoscopy" OR "Colonoscopies" OR "Colonoscopic Surgical Procedures" OR "Colonoscopic Surgical Procedure" OR "Procedure, Colonoscopic Surgical" OR "Procedures, Colonoscopic Surgical" OR "Surgical Procedure, Colonoscopic" OR "Surgery, Colonoscopic" OR 
"Surgical Procedures, Colonoscopic" OR "Colonoscopic Surgery" OR "Colonoscopic Surgeries" OR "Surgeries, Colonoscopic" AND "Cathartics" OR "Bowel preparation" OR "Bowel Evacuants" OR "Evacuants, Bowel" OR "Purgatives" OR "Bowel Preparation Solutions" OR "Preparation Solutions, Bowel" OR "Solutions, Bowel Preparation". The reference lists of the retrieved studies and previous reviews and meta-analyses were manually searched for potential RCTs. An information specialist reviewed the search strategies.

\section{Data extraction}

Two reviewers independently extracted data on interventions and outcomes. They also recorded study and patient characteristics including first author, year of publication, study location, target population, intervention of single versus split dose group, main outcomes, and conclusions. They compared results and resolved disagreements by discussion with a third party. Authors were contacted to clarify ambiguities and to request data on outcomes missing in primary reports.

\section{Quality assessment}

The two authors independently assessed the risk of bias in the included RCTs. Reviewers evaluated the following items for each RCT: sequence generation, allocation hiding, patient and personnel blindness, result evaluator blindness, incomplete result data, and selective reporting and other issues. According to the judgment criteria, each part can be classified as low, high, or unclear risk.

\section{Data synthesis and analysis}

The extracted data were processed and analyzed with Review Manager (version 5.3) (18). All data were independently extracted and carefully checked by the two authors. Binary outcomes were showed as Mantel-Haenszelstyle odds ratios (ORs) with 95\% confidence intervals (CIs). Continuous outcomes were indicated as mean differences (MDs). And fixed-effect model was used in the case of homogeneity $\left(\mathrm{P}>0.10\right.$ and $\left.\mathrm{I}^{2}<50 \%\right)$. And random-effect model was conducted in the case of significant heterogeneity $(\mathrm{P}>0.10$ and $\mathrm{I}^{2} \geq 50 \%$ ). If there was significant heterogeneity, we tried to perform subgroup analysis and further analyzed the source of heterogeneity in RCT. The publication bias was assessed by a funnel plot and asymmetry was checked by performing an Egger regression test. Differences with $\mathrm{P}<0.1$ were considered as being statistically significant.

\section{Results}

\section{Screening}

The electronic searches resulted in 96 unique citations (Figure 1). After reference and full-text screening, after screening the titles and abstracts of the citations, we included 17 potentially related studies for another round of full-text review. These studies were further screened and reviewed. Finally, four studies $(5,19-21)$ were included in this present meta-analysis.

\section{Included studies}

The four included studies that compared 249 pediatric patients, comprising 106 patients who underwent single dose of PEG and 143 patients who underwent split dose of PEG. All of the four RCTs were conducted in different countries, namely, Iran, India, Thailand, and the USA (Table 1).

\section{Risk of bias assessment}

Figures 2,3 demonstrate the risk of bias of included in the four RCTs. Although all of the RCTs mentioned the randomization, the method of randomization of one RCT Konanki 2010 remained unclear, meanwhile none reported allocation blinding. Given the nature of interventions, informed consent was taken from either parent of all children, blinding participants and personnel is impossible. No study reported the blinding design on participants and personnel. On the outcome assessment process, all the trails reported that the attending staffs were blinded to the preparation regimen which were considered as low risk of bias. Reporting bias was not detected.

\section{Outcome analysis}

As the trial's populations and interventions were judged in high similarity, four studies included in data synthesis were eligible for meta-analysis, resulting in a total of 356 participants. This review used fixed-effect to produce an 


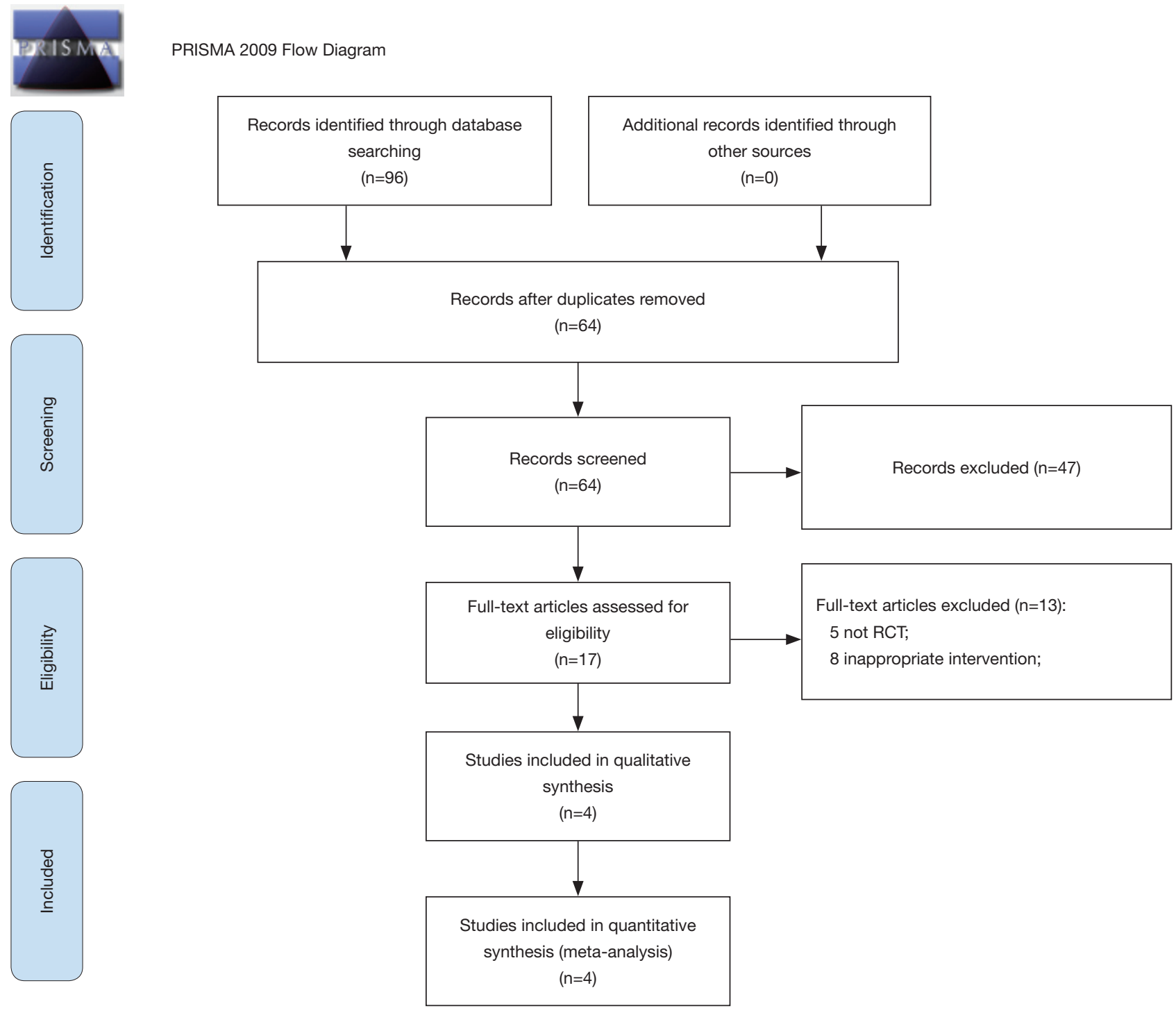

Figure 1 Flow chart of study selection. RCT, randomized controlled trial.

overall summary of included trials.

\section{Efficacy of bowel preparation}

All the trails reported the comparisons of efficacy between single- versus split-dose regimen. The summary OR on the satisfactory preparation was 0.36 (95\% CI: -0.12 to 1.10$)$, and heterogeneity was evident $\left(\mathrm{P}=0.03, \mathrm{I}^{2}=72 \%\right)$ (Figure 4).

\section{Acceptability}

All the trails reported the acceptability of the two regimens.
The summary OR on the acceptability was $0.50(95 \%$ CI: 0.29 to 0.85$)$, and heterogeneity was evident $(\mathrm{P}=0.92$, $\mathrm{I}^{2}=0 \%$ ) (Figure 5).

\section{Adverse effects}

All the trails reported the adverse effects of the two regimens including nausea, abdominal pain. The summary OR on the incidence of nausea was 2.10 (95\% CI: 1.29 to 3.42 ), and heterogeneity was evident $\left(\mathrm{P}=0.35, \mathrm{I}^{2}=9 \%\right)$ (Figure 6). The summary OR on the incidence of abdominal pain was 1.39 (95\% CI: 0.67 to 2.89 ), and heterogeneity was evident 
Table 1 The characteristics of included RCTs

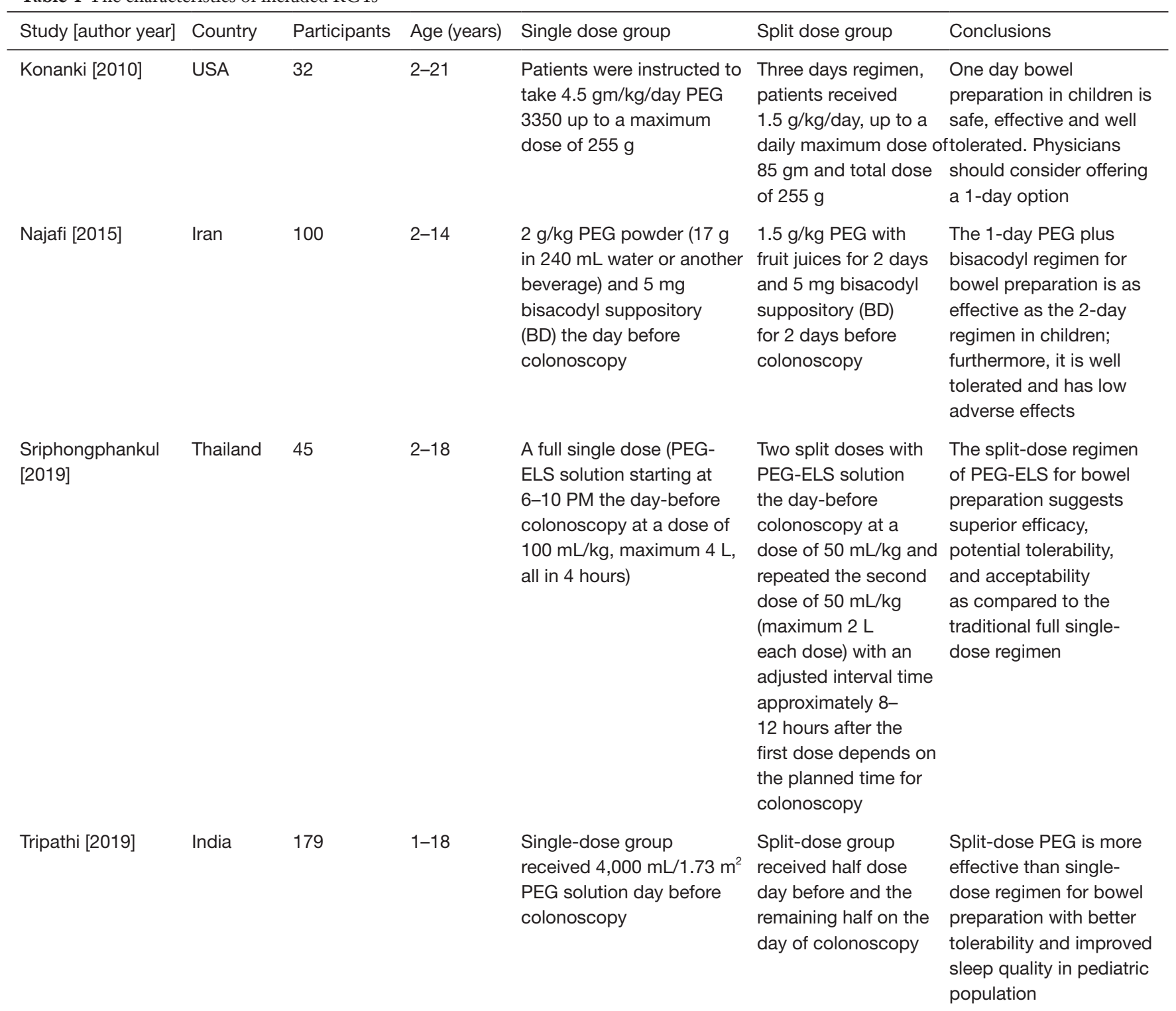

RCT, randomized controlled trial; PEG, polyethylene glycol; ELS, electrolyte solution.

$\left(\mathrm{P}=0.51, \mathrm{I}^{2}=0 \%\right)$ (Figure 7$)$.

\section{Subgroup and sensitivity analyses}

We did not conduct subgroup analyses in this study because of the small heterogeneity and limited number of the included RCTs. We attempted to evaluate publication bias by a funnel plot. However, publication bias was not determined due to the limited number of the included RCTs (less than 10). Sensitivity analyses are used to investigate the influence of one study on the overall risk estimate by removing one study in each turn for every included result. The results suggested that the overall risk estimates were not substantially changed by any single study.

\section{Discussion}

To take single or split dose polyethylene glycol for bowel preparation in children undergoing colonoscopy is an important question that warrants discrete verification. The 


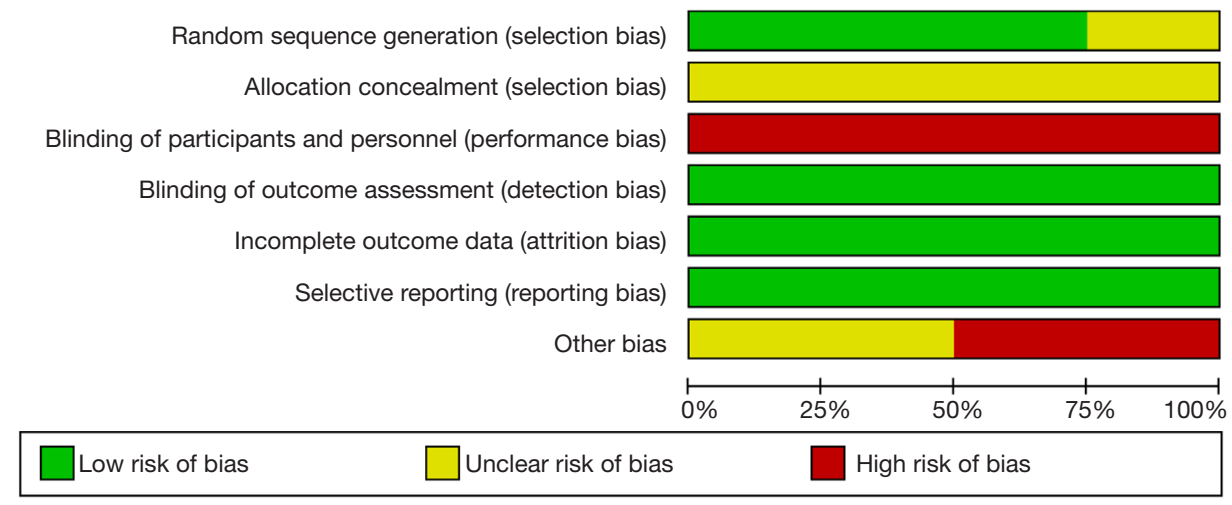

Figure 2 Risk of bias graph.

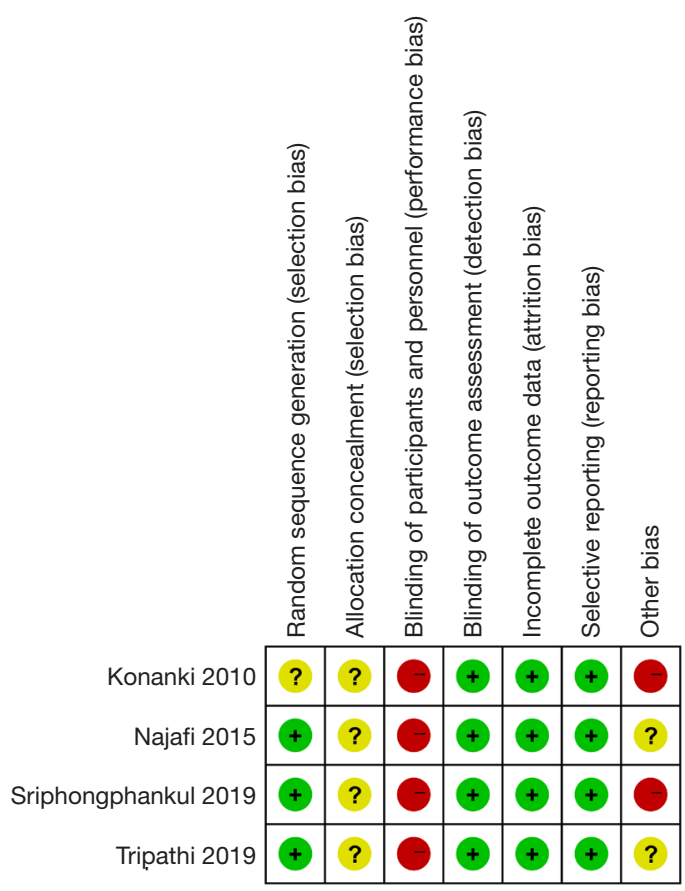

Figure 3 Risk of bias summary.

purpose of the current systematic review was to summarize available evidence to the use of PEG for colonic preparation in pediatric population. The principal conclusions confirm that use of split-dose regimens results in greater proportions of children with adequate preparations. However, due to the small sample sizes of some studies (45 and 32) and the lack of demographic data specifically assessing the children who underwent colonoscopy, we failed to address any lack of generality and possible selection bias in the summary findings. However, we noted some statistical heterogeneity, which indicates inconsistencies in the study results, proving many possible explanations for preplanned sensitivity analyses and meta-regression searches. Alterations in clinical protocol (e.g., PEG and PEG 3350 may differ in efficacy), as well as the presence of two different dosedefined regiments and the main definition of intestinal cleanliness, it may explain these differences in observation and statistical results.

Currently, electrolyte-free PEG is the mainstay of children with constipation. It has proven to be effective, safe, tasty and has excellent compliance (22). Due to these characteristics, the PEG as a choice of enteric preparation has been studied. PEG is considered an effective intestinal cleansing regimen and is associated with less electrolyte imbalance $(23,24)$. Several studies $(14,25-27)$ have demonstrated that PEG is superior or equal on efficacy of bowel preparation comparing with other regimens (senna, $\mathrm{NaPico}$ with magnesium citrate, sodium phosphate) among pediatric populations. Both senna and sodium phosphate can cause side effects, such as dehydration, which lead extra fluids intake to the patients. Furthermore, previous studies $(9,28)$ have shown that PEG can be used as a safe and effective formulation for children at a dose of $1.5 \mathrm{~g} / \mathrm{kg}$ for 4 days. However, it has been reported that bowel preparation should be ideally completed in a shorter period of time (12). Several other intestinal preparation therapies have been used for colonoscopy in children. Traditionally, PEG and electrolyte lavage fluids have been used in children with satisfactory results. However, its acceptability and tolerance are limited due to its salty taste and the large volume, the insertion nasogastric tube that is often required for children (29). Another commonly used effective formulation is sodium 


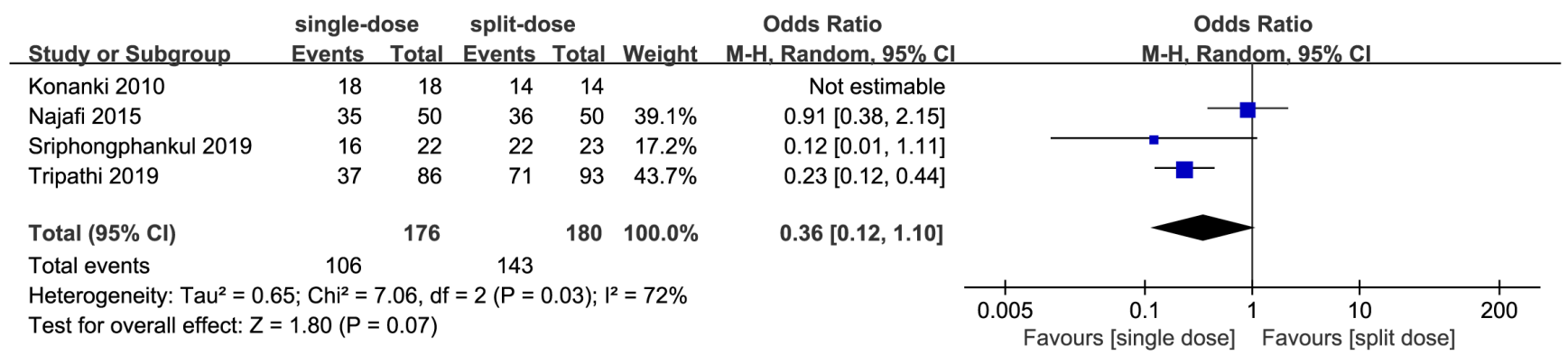

Figure 4 The forest plot for the efficacy of bowel preparation. CI, confidence interval.

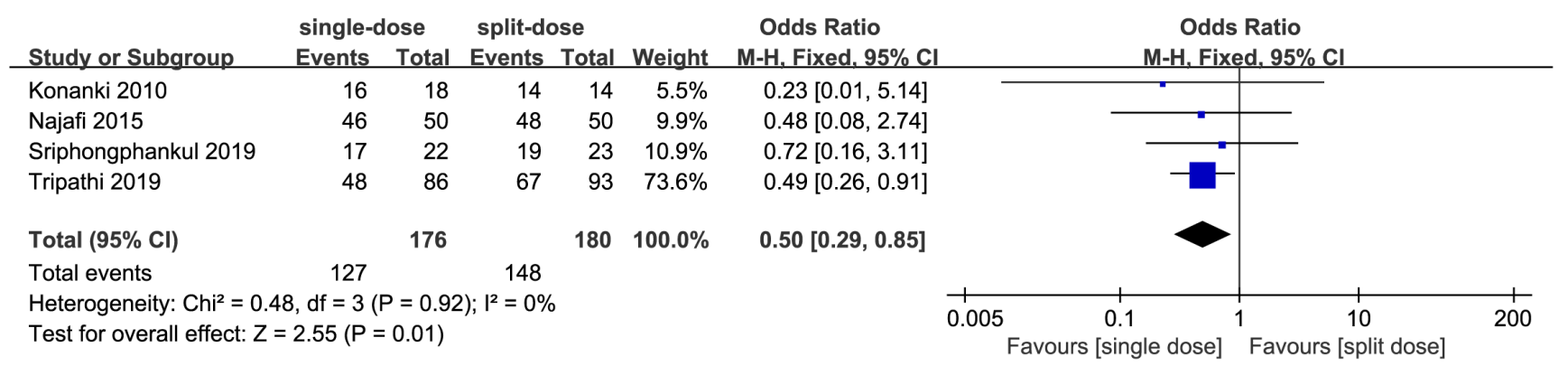

Figure 5 The forest plot for the acceptability. CI, confidence interval.

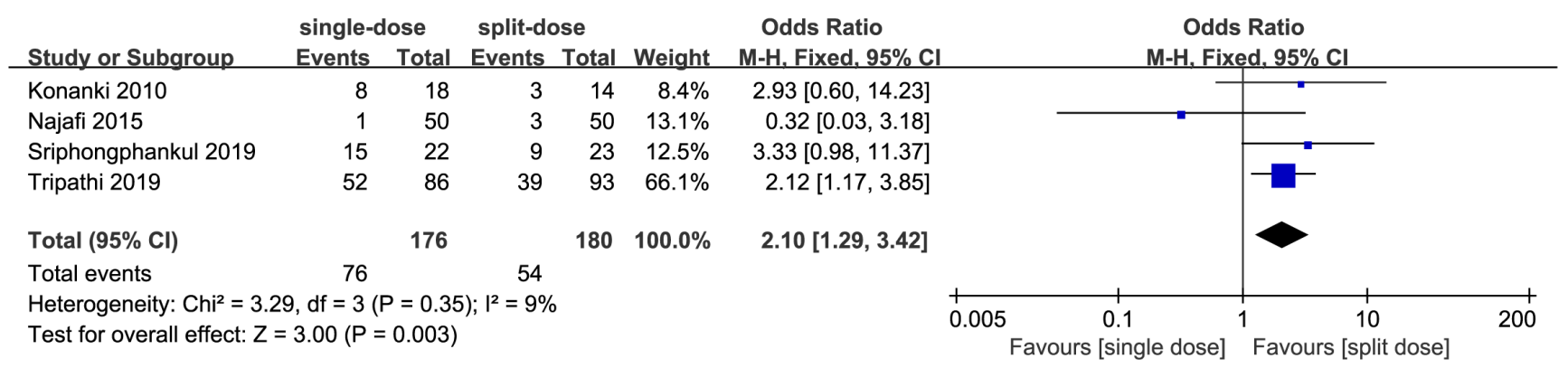

Figure 6 The forest plot for the incidence of nausea. CI, confidence interval.

phosphate, however, it may cause electrolyte abnormalities, such as severe hyperphosphatemia and hypocalcemia (30). Besides, it can lead to colonic mucosal changes that mimic inflammatory bowel disease (31). Another bowel preparation therapies combining different drugs (such as magnesium citrate, bisacodyl, relaxants et al.) have shown variable efficacy and adverse effects (32,33). Previous study (34) has reported the efficacy and safety of a 1-day PEG bowel preparation protocol for colonoscopy in children, but they have not use standard scoring systems to assess outcomes such as tolerance and adequacy of the bowel preparation. In addition, children must drink a large amount of PEG solution (1.9 liters) with a commercially available sports drink within 2 hours. The results of our synthesized analysis have indicated that split-dosed PEG regimen provide excellent acceptance and efficacy without major adverse effects. Furthermore, the present regimen can be used during the period of weekend, resulting in children to avoid 


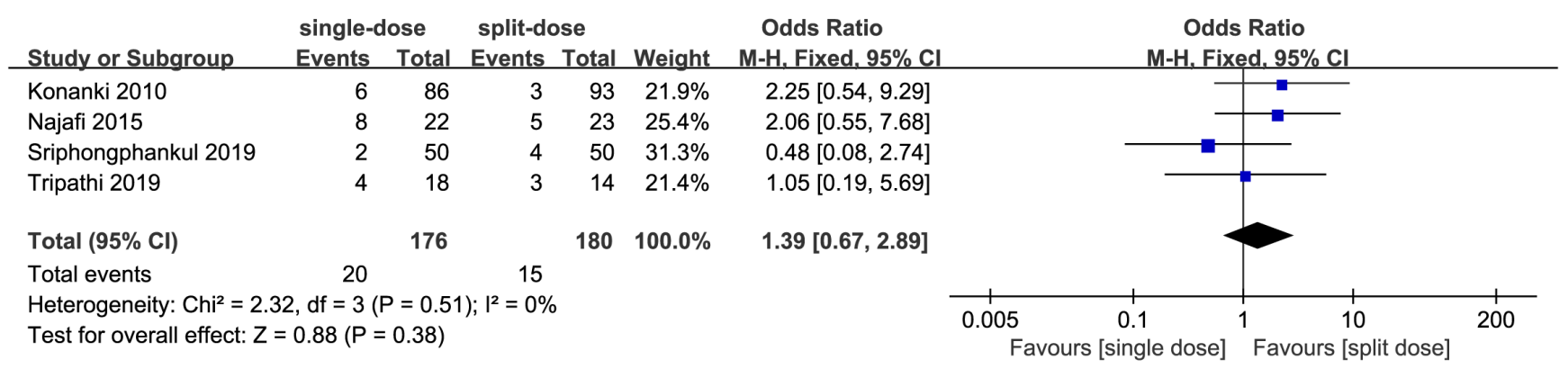

Figure 7 The forest plot for the incidence of abdominal pain. CI, confidence interval.

unnecessary loss of school days.

For the colonoscopy procedure, there are some concerns with taking one dose or split dose of the bowel preparation solution. For example, the bowel movements during the transfer to the endoscopic unit may cause inconvenience to children. A randomized study comparing split and singledose bowel preparations found that patients in the split-dose group had more toilet stops on their way to the hospital (35). However, the patients in the split-dose group found easier completion of bowel preparation with more satisfaction compared to the single-dose group. Another concern is that the bowel preparation solution is inhaled from the stomach into the lungs after sedation. However, previous study has found that no difference in residual gastric volume between patients who fasted for $2 \mathrm{~h}$ and those who fasted for $6-23 \mathrm{~h}$ $(36,37)$. Ideal bowel preparation should be effective, safe and easily accepted by children (38). PEG seems to meet these requirements (39). However, the appropriate duration and dose need to be determined by further RCTs.

Several limitations must be considered in this study. Firstly, synthesis was severely limited due to the heterogeneity of clinical and methodological methods, which was reflected in less research in meta-analysis. Secondly, the blind method and allocation design in the included RCTs remained unclear, and the nature of bowel preparation makes it difficult to perform blind methods. Future research should focus more on improving the study design to make the results more reliable. Thirdly, the assessment of satisfactory preparation and acceptability in the included RCTs was still quite subjective, and there might be significant deviations, so it is necessary to conduct future studies on those indicators with standardized scales.

\section{Conclusions}

Taking all the evidence together, split-dose PEG is more effective than single-dose for children underwent colonoscopy. Considering the small sample size of the included studies and the inconsistent evaluation tools. Future studies should be conducted in multiple centers to expand the sample size and with unified tools so as to summarize the research results as well as to obtain stronger evidence.

\section{Acknowledgments}

Funding: None.

\section{Footnote}

Reporting Checklist: The authors have completed the PRISMA reporting checklist. Available at http://dx.doi.org/10.21037/ apm-20-582

Conflicts of Interest: All authors have completed the ICMJE uniform disclosure form (available at http://dx.doi. org/10.21037/apm-20-582). The authors have no conflicts of interest to declare.

Ethical Statement: The authors are accountable for all aspects of the work in ensuring that questions related to the accuracy or integrity of any part of the work are appropriately investigated and resolved. Since our 
manuscript is a meta-analysis, no ethical permissions were needed. The study conformed to the provisions of the Declaration of Helsinki (as revised in 2013).

Open Access Statement: This is an Open Access article distributed in accordance with the Creative Commons Attribution-NonCommercial-NoDerivs 4.0 International License (CC BY-NC-ND 4.0), which permits the noncommercial replication and distribution of the article with the strict proviso that no changes or edits are made and the original work is properly cited (including links to both the formal publication through the relevant DOI and the license). See: https://creativecommons.org/licenses/by-nc-nd/4.0/.

\section{References}

1. Rex DK, Schoenfeld PS, Cohen J, et al. Quality indicators for colonoscopy. Gastrointest Endosc 2015;81:31-53.

2. Halpern Z, Gross SA, Gralnek IM, et al. Comparison of adenoma detection and miss rates between a novel balloon colonoscope and standard colonoscopy: a randomized tandem study. Endoscopy 2015;47:238-44.

3. Saltzman JR, Cash BD, Pasha SF, et al. Bowel preparation before colonoscopy. Gastrointest Endosc 2015;81:781-94.

4. Liu X, Luo H, Zhang L, et al. Telephone-based reeducation on the day before colonoscopy improves the quality of bowel preparation and the polyp detection rate: a prospective, colonoscopist-blinded, randomised, controlled study. Gut 2014;63:125-30.

5. Sorser SA, Konanki V, Hursh A, et al. 1-day bowel preparation with polyethylene glycol 3350 is as effective and safe as a 3-day preparation for colonoscopy in children. BMC Res Notes 2014;7:648.

6. Lorenzo-Zúñiga V, Moreno de Vega V, Marín I, et al. Improving the quality of colonoscopy bowel preparation using a smart phone application: a randomized trial. Dig Endosc 2015;27:590-5.

7. Voiosu T, Voiosu A, Voiosu R. Bowel preparation for colonoscopy: state of the art. Curr Opin Gastroenterol 2016;32:385-6.

8. Gordon M, Karlsen F, Isaji S, et al. Bowel preparation for elective procedures in children: a systematic review and meta-analysis. BMJ Paediatr Open 2017;1:e000118.

9. Pashankar DS, Uc A, Bishop WP. Polyethylene glycol 3350 without electrolytes: a new safe, effective, and palatable bowel preparation for colonoscopy in children. J Pediatr 2004;144:358-62.

10. Safder S, Demintieva Y, Rewalt M, et al. Stool consistency and stool frequency are excellent clinical markers for adequate colon preparation after polyethylene glycol 3350 cleansing protocol: a prospective clinical study in children. Gastrointest Endosc 2008;68:1131-5.

11. Mytyk A, Lazowska-Przeorek I, Karolewska-Bochenek K, et al. Clear liquid versus low-fibre diet in bowel cleansing for colonoscopy in children: a randomized trial. J Pediatr Gastroenterol Nutr 2018;66:720-4.

12. Pall H, Zacur GM, Kramer RE, et al. Bowel preparation for pediatric colonoscopy: report of the NASPGHAN endoscopy and procedures committee. J Pediatr Gastroenterol Nutr 2014;59:409-16.

13. Sahn B, Chen-Lim ML, Ciavardone D, et al. Safety of a 1-day polyethylene glycol 3350 bowel preparation for colonoscopy in children. J Pediatr Gastroenterol Nutr 2016;63:19-24.

14. Vejzovic V, Wennick A, Idvall E, et al. Polyethylene glycolor sodium picosulphate-based laxatives before colonoscopy in children. J Pediatr Gastroenterol Nutr 2016;62:414-9.

15. Samarasena JB, Muthusamy VR, Jamal MM. Split-dosed MiraLAX/Gatorade is an effective, safe, and tolerable option for bowel preparation in low-risk patients: a randomized controlled study. Am J Gastroenterol 2012;107:1036-42.

16. Aoun E, Abdul-Baki H, Azar C, et al. A randomized singleblind trial of split-dose PEG-electrolyte solution without dietary restriction compared with whole dose PEGelectrolyte solution with dietary restriction for colonoscopy preparation. Gastrointest Endosc 2005;62:213-8.

17. Martel M, Barkun AN, Menard C, et al. Split-Dose Preparations Are Superior to Day-Before Bowel Cleansing Regimens: A Meta-analysis. Gastroenterology. 2015;149:79-88.

18. Wen Z, Xie A, Peng M, et al. Is discard better than return gastric residual aspirates: a systematic review and metaanalysis. BMC Gastroenterol 2019;19:113.

19. Sriphongphankul H, Tanpowpong P, Lertudomphonwanit $\mathrm{C}$, et al. Split dose versus full single-dose regimen of polyethylene glycol for bowel preparation in pediatric colonoscopy: a pilot study of randomized controlled trial. Eur J Gastroenterol Hepatol 2019;31:1382-6.

20. Najafi M, Fallahi GH, Motamed F, et al. Comparison of one and two-day bowel preparation with polyethylene glycol in pediatric colonoscopy. Turk J Gastroenterol 2015;26:232-5.

21. Tripathi PR, Poddar U, Yachha SK, et al. Efficacy of single- versus split-dose polyethylene glycol for colonic preparation in children: a randomized control study. J 
Pediatr Gastroenterol Nutr 2020;70:e1-6.

22. Yoo T, Bae SH. Efficacy and safety of combined oral and enema therapy using polyethylene glycol 3350-electrolyte for disimpaction in pediatric constipation. Pediatr Gastroenterol Hepatol Nutr 2017;20:244-51.

23. Mathus-Vliegen E, Pellisé M, Heresbach D, et al. Consensus guidelines for the use of bowel preparation prior to colonic diagnostic procedures: colonoscopy and small bowel video capsule endoscopy. Curr Med Res Opin 2013;29:931-45.

24. Kumar A, Hussain A. Preoperative bowel preparation in children: polyethylene glycol versus normal saline. Afr J Paediatr Surg 2013;10:235-8.

25. Terry NA, Chen-Lim ML, Ely E, et al. Polyethylene glycol powder solution versus senna for bowel preparation for colonoscopy in children. J Pediatr Gastroenterol Nutr 2013;56:215-9.

26. Di Nardo G, Aloi M, Cucchiara S, et al. Bowel preparations for colonoscopy: an RCT. Pediatrics 2014;134:249-56.

27. Dehghani SM, Javaherizadeh H, Haghighat M, et al. Bowel preparation before colonoscopy for children: comparison of efficacy of three different methods. Arq Gastroenterol 2015;52:311-4.

28. Kumar AS, Beutler BL, Attard TM. One-day oral polyethylene glycol based cleanout is effective for precolonoscopy preparation in children. BMC Gastroenterol 2018;18:170.

29. Phatak UP, Johnson S, Husain SZ, et al. Two-day bowel preparation with polyethylene glycol 3350 and bisacodyl: a new, safe, and effective regimen for colonoscopy in children. J Pediatr Gastroenterol Nutr 2011;53:71-4.

30. Lee SW, Bang CS, Park TY, et al. Split-dose bowel preparation for colonoscopy: 2 liters polyethylene glycol with ascorbic acid versus sodium picosulfate versus oral sodium phosphate tablets. Korean J Gastroenterol

Cite this article as: Cao J, Zhang W, Hu J, Huang Y, Zhao L, Cai R, Bao Y, Li M. Single versus split dose polyethylene glycol for bowel preparation in children undergoing colonoscopy: a systematic review and meta-analysis. Ann Palliat Med 2020;9(5):3028-3037. doi: 10.21037/apm-20-582
2017;70:89-95.

31. Cheng J, Tao K, Shuai X, et al. Sodium phosphate versus polyethylene glycol for colonoscopy bowel preparation: an updated meta-analysis of randomized controlled trials. Surg Endosc 2016;30:4033-41.

32. Shaoul R, Haloon L. An assessment of bisacodylbased bowel preparation for colonoscopy in children. J Gastroenterol 2007;42:26-8.

33. Berger T, Classen M, Engelhardt H, et al. Bowel preparation in pediatric colonoscopy: results of an open observational study. Endosc Int Open 2016;4:E820-7.

34. Adamiak T, Altaf M, Jensen MK, et al. One-day bowel preparation with polyethylene glycol 3350: an effective regimen for colonoscopy in children. Gastrointest Endosc 2010;71:573-7.

35. Parra-Blanco A, Nicolas-Perez D, Gimeno-Garcia A, et al. The timing of bowel preparation before colonoscopy determines the quality of cleansing, and is a significant factor contributing to the detection of flat lesions: a randomized study. World J Gastroenterol 2006;12:6161-6.

36. Phillips S, Hutchinson S, Davidson T. Preoperative drinking does not affect gastric contents. Br J Anaesth 1993;70:6-9.

37. Schmidt AR, Buehler KP, Both C, et al. Liberal fluid fasting: impact on gastric $\mathrm{pH}$ and residual volume in healthy children undergoing general anaesthesia for elective surgery. Br J Anaesth 2018;121:647-55.

38. Katelaris P, Naganathan V, Liu K, et al. Comparison of the effectiveness of polyethylene glycol with and without electrolytes in constipation: a systematic review and network meta-analysis. BMC Gastroenterol 2016;16:42.

39. Kherad O, Restellini S, Martel M, et al. Polyethylene glycol versus sodium picosulfalte bowel preparation in the setting of a colorectal cancer screening program. Can J Gastroenterol Hepatol 2015;29:384-90. 